\title{
Tibiakopffraktur beim alten Menschen
}

\author{
$\square$ Christian Frank, Paul Alfred Grützner, Andreas Wentzensen
}

\section{Zusammenfassung}

Alterstraumatologie kann man unter den Stichworten Schenkelhalsfraktur, osteoporotischer Wirbelbruch, Oberarmkopfbruch und Handgelenksbruch plakativ zusammenfassen. Die Überalterung in unserer Gesellschaft wird aber nicht nur zu einer realen Zunahme dieser Brüche führen. Es zeigt sich bereits jetzt, dass andere Verletzungen wie etwa der Schienbeinkopfbruch in dieser Altersgruppe ebenfalls zunehmen. Allerdings dominieren hier leichtere Unfälle wie Fahrradsturz oder Sturz beim Laufen. Rasanztraumen treten selten auf. Bei der Versorgung dieser Frakturen wird den Begleiterkrankungen eine wichtige Bedeutung zukommen. Die Erfahrungen in dieser Richtung sind noch gering, es können aber Analogieschlüsse zu den
Schenkelhalsfrakturen gezogen werden. Welche Auswirkung das Risikoprofil des Einzelnen bei der Behandlung der Schienbeinkopfbrüche hat, ist unklar. Sie stellen aber eine besondere Herausforderung bei der operativen und perioperativen Versorgung dar. Sie beeinflussen die Operationstechnik, den Zugang und die Wahl des Implantats. Sie können die stationäre Aufenthaltsdauer verlängern und beeinträchtigen die Rehabilitation. Das Behandlungsziel beim Jungen wie beim Alten ist aber das Gleiche. Gewünscht ist eine rasche Rückkehr in die persönliche Umgebung mit guter Funktion und belastbarer Extremität. Dies erfordert u.a. eine möglichst anatomische Rekonstruktion der Gelenkfläche. Es bedeutet daher auch eine Ausweitung der Operationsindikation in der Gruppe der Patienten.

\section{Einleitung}

Immer mehr Menschen werden immer älter [9]. Im Gleichschritt mit der Überalterung unserer Gesellschaft nimmt die Zahl der Verletzten über 70 in den letzten Jahren spürbar zu.

Einerseits, weil durch medizinischen Fortschritt ein hohes Aktivitätsniveau auch in der Gruppe der Senioren besteht. Andererseits, weil es durch die typischen Begleiterkrankungen zur erhöhten Sturzanfälligkeit kommt und das Frakturrisiko zunimmt.

Die Rüstigeren bringen sicher bessere Voraussetzungen für die Frakturversorgung mit, haben aber auch höhere Ansprüche an das postoperative Ergebnis.

OP-JOURNAL 2005; 21: 180-185

(c) Georg Thieme Verlag KG Stuttgart · New York

Die andere, mit zunehmendem Lebensalter zahlenmäßig gewichtigere Gruppe sind die multimorbiden Hinfälligen. Hier findet man regelhaft mehrere der folgenden Begleiterkrankungen: eine eingeschränkte Koordination, reduzierte geistige Fähigkeiten bis zur Demenz, Herz-, Lungen- und Nierenerkrankungen sowie den Alltag beeinträchtigende Veränderungen des zentralen Nervensystems. Zudem können Adipositas, Stoffwechselerkrankungen wie Diabetes, Gefäßerkrankungen und Gerinnungsstörungen vorliegen. Herzinfarkte oder Schlaganfälle stellen eine weitere Belastung dar. Man findet eine altersbedingte Abnahme der Leistungsfähigkeit aller Organe, der Muskeln und des Skelettsystemes. Psychiatrische Störungen wie Altersdepressionen oder akute Durchgangssyndrome werden beobachtet [6]. Eine ganz wichtige Rolle spielt in dieser Altersgruppe die Osteoporose, auch bei Männern!
H.R. Siebert und A. Beck sprechen in diesem Zusammenhang vom Syndrom des Verletzten im höheren Lebensalter [10].

Der Einfluss dieser Risikofaktoren auf das Operationsergebnis und die Wahl des Operationsverfahrens ist bei hüftgelenksnahen Oberschenkelbrüchen anerkannt. Treten Brüche an anderen Skelettabschnitten auf, ergibt sich ihre Bedeutung im Rahmen der Zugangsmorbidität und im Hinblick auf die anstehende Rehabilitation bzw. das perioperative Management im Allgemeinen.

Bei gleich bleibender Bevölkerungszahl wird es in den kommenden Jahren mehr über Sechzigjährige geben, als Patienten im arbeitsfähigen Alter [9].

Zwangsläufig wird in Zukunft das Kollektiv der alten Patienten größer. Damit wird auch die Zahl der Brüche steigen, die man bisher überwiegend bei Jüngeren vorgefunden hat. Untersuchungen belegen dies bereits für den Schienbeinkopfbruch [2]. Es handelt sich hierbei zwar nicht um eine typische Fraktur des geriatrischen Patienten, dennoch zeigt die Auswertung der zugrunde liegenden Unfallmechanismen und der Geschlechterverteilung den Einfluss des Alters und der Osteoporose. Es genügt bei schlechter Knochenqualität ein vergleichsweise leichtes Unfallereignis als Frakturauslöser [4].

Die klinische Erfahrung zeigt, dass die in der Alterstraumatologie gewonnenen Erkenntnisse analog übertragen werden können. Weichteilunterblutungen im Frakturbereich oder das Vorliegen einer Durchblutungsstörung müssen bei der Wahl der Versorgung und des Operationszeitpunktes berücksichtigt werden. Abhängig von der Knochenqualität ist mit verzögerter Knochenbruchheilung und sekundärem Korrekturverlust $\mathrm{zu}$ rechnen. Die Begleiterkrankungen werden Einfluss auf die Rehabilitationsfähigkeit und damit auf die Dauer des stationären Aufenthaltes haben (Abb.1). 

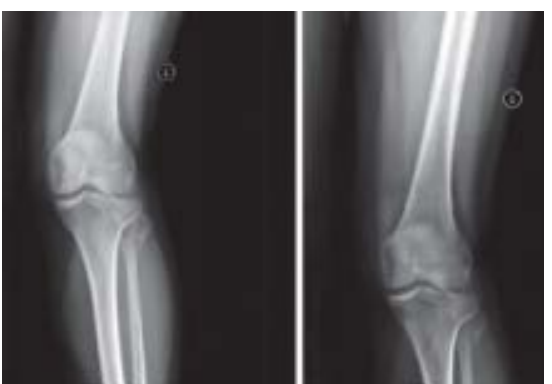

Abb.1 Unfallbild einer 70-Jährigen nach Fahrradsturz. Begleitend bestand ein leichtes Schädel-Hirn-Trauma. An Grunderkrankungen lag eine kompensierte Herzinsuffizienz mit Belastungsdyspnoe vor, außerdem eine Blasenschwäche. In der postoperativen Phase Mobilisation mit Gehbock und Gehwagen, eine Teilbelastung konnte wegen Koordinationsschwäche nicht umgesetzt werden.

\section{Klassifikation}

Die weiteste Verbreitung hat die AO-Klassifikation (Abb.2). Am anspruchvollsten zu behandeln sind die Frakturen mit Gelenkbeteiligung (Abb.3). Im eigenen Krankengut wurden meistens Spaltimpressionsbrüche des lateralen Tibiaplateaus versorgt.
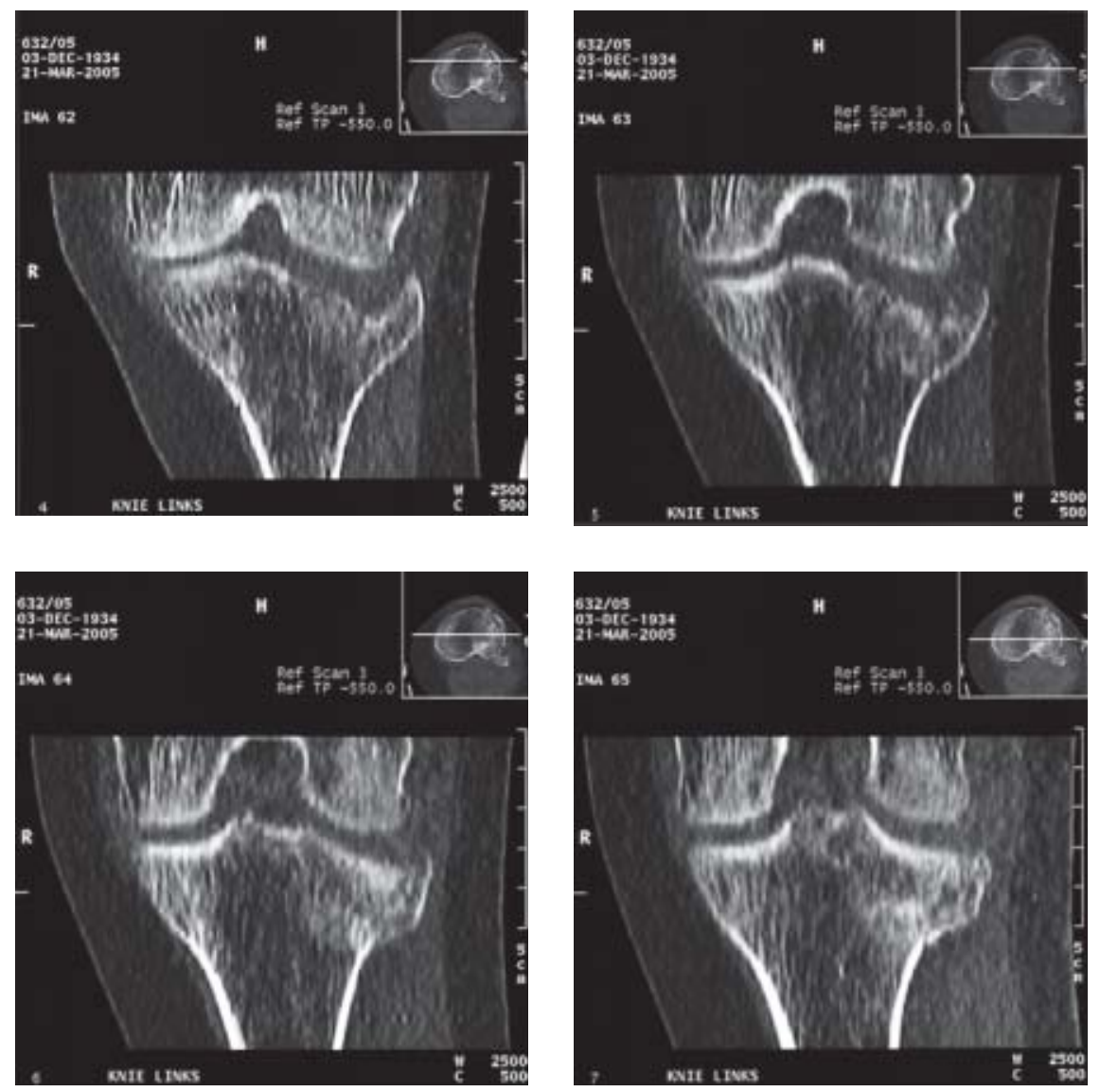

Abb.3 Das präoperative CT zur Operationsplanung der Patientin aus Abb.1. Es besteht eine Impression der lateralen Gelenkfläche mit Trümmerzone.

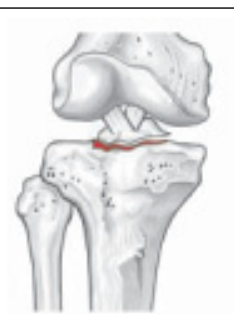

A1

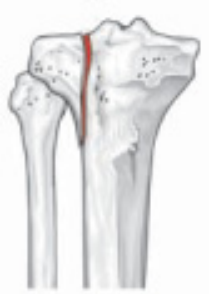

B1

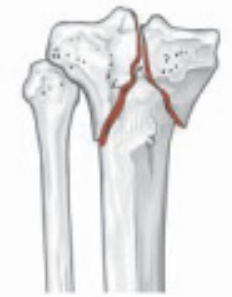

C1

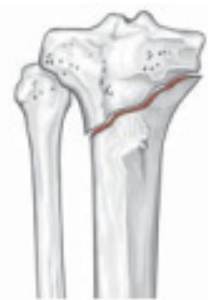

A2

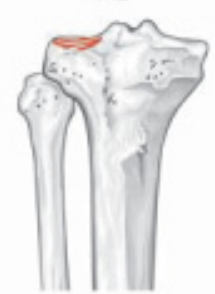

B2

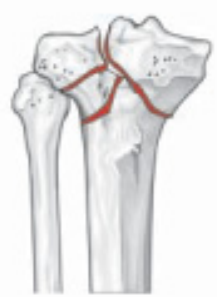

C2

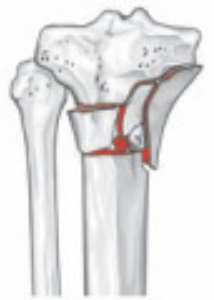

A3

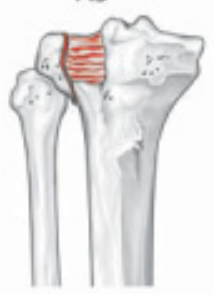

B3

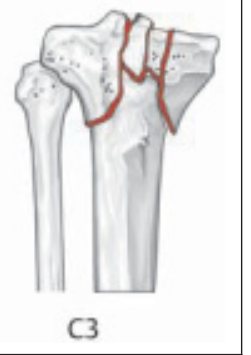

Abb. 2 AO-Klassifikation der proximalen Tibiafrakturen aus [3]

\section{Tibia, Fibula}

41 Proximal

A - extraartikuläre Fraktur A1 Ausriss

A2 metaphysär einfach A3 metaphysär mehrfragmentär

\section{B - partielle Gelenkfraktur} B1 reine Spaltung

B2 reine Impression

B3 Impression und Spaltung

\section{C - vollständige Gelenk-} fraktur

C1 artikulär einfach, metaphysär einfach

C2 artikulär einfach, metaphysär mehrfragmentär

C3 mehrfachfragmentär

Die Osteosynthese des Schienbeinkopfbruches muss beim alten Patienten mit dem gleichen Anspruch wie beim jüngeren durchgeführt werden. Nur dann kann sie zu vergleichbaren Ergebnissen führen.

Das erfordert eine möglichst anatomische Gelenkflächenrekonstruktion, eventuell mit Spongiosaunterfütterung (Abb.4). Ziel ist die übungsstabile Versorgung. An- 
gestrebt wird ein voll belastbares, stabiles und gut bewegliches Kniegelenk.

Das bedeutet, dass die Indikation zu operativen Versorgung auch beim alten, mobilen Patienten eher weit gefasst wird.

Es steht die rasche Wiederherstellung der Funktion und Mobilität zur Vermeidung von Hospitalisationsschäden im Vordergrund. Das beinhaltet eine früh einsetzende physiotherapeutische Übungsbehandlung. Nicht selten müssen Internisten und Neurologen in die Behandlung miteingebunden werden.

\section{OP-Prinzip}

Zumeist wird eine offene Reposition zur Wiederherstellung der Gelenkfläche vorgenommen. Seltener kommen arthroskopisch gestützte Schraubenosteosynthesen oder Operationsverfahren mit Navigation zum Einsatz. Da osteoporotische Frakturen mit ausgedehnten Trümmerzonen einhergehen, spielt die Auswahl des Implantats eine entscheidende Rolle. Der Einsatz winkelstabiler Osteosyntheseverfahren ermöglicht die feste Verankerung der Schraubenköpfe in den Plattenlöchern, womit man dem Problem der Implantatlockerung begegnet. Die Schrauben tragen dann wie eine Balkenkonstruktion die Gelenkfläche.

Bei subchondralen Trümmerzonen mit Substanzverlust muss eine Spongiosaplastik erwogen werden.

Ein Problem stellt hier jedoch die oft schlechte Spongiosaqualität beim älteren Patienten dar. Beschrieben wird deshalb der Einsatz von Knochenersatzmaterialien.

\section{OP-Zeitpunkt}

Die Wahl des Operationszeitpunktes hängt von den begleitenden Weichteilverletzungen $a b$.

Ein manifestes Kompartmentsyndrom zwingt zum sofortigen Handeln. Im Rahmen der Kompartmentspaltung kann dann die primäre Osteosynthese mit temporärer Weichteildeckung mittels Vakuumversiegelung erfolgen. Sekundär wird schließlich der Wundverschluss an die bestehende Weichteilsituation angepasst.

Auch offene Verletzungen eventuell mit Gefäß- und Nervenbeteiligung müssen

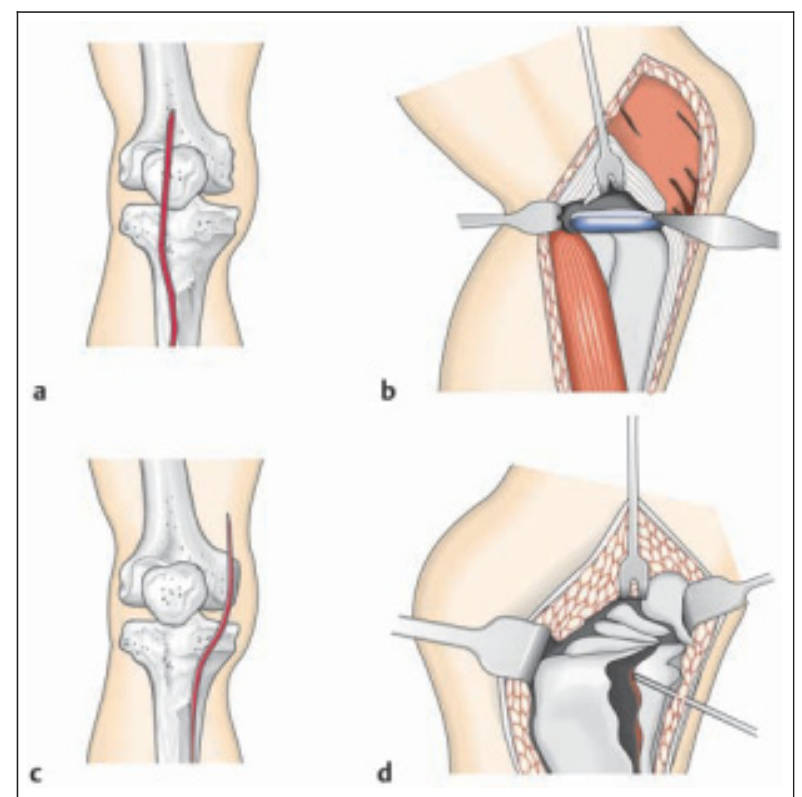

Abb. 5 Zugangswege zum Schienbeinkopf aus [3]. Medianer (a, b), medialer oder lateraler Zugang (c, d). Gelenkeröffnung unterhalb des Meniskus.

sofort operativ versorgt werden, Gefäßverletzungen der Arteria poplitea haben absolute Priorität. Solch schwere Verletzungen sind aber in dieser Altersgruppe selten. Zur primären Stabilisierung kann bei ausgedehnten Weichteilschäden bis zur sekundären Versorgung ein gelenküberbrückender Fixateur angelegt werden. Ansonsten kann man die Konsolidierung der Weichteile abwarten und die Zeit zur Mitbehandlung der Begleiterkrankungen und zur Ermittlung der Sturzursache nutzen.

\section{OP-Indikation}

Entsprechende Weichteile vorausgesetzt, werden alle instabilen, nicht reponierbaren Brüche und solche mit mehr als zwei Millimeter messenden Gelenkstufen offen versorgt.

\section{Kontraindikationen}

Als Kontraindikationen gelten ein schlechter Allgemeinzustand und lebensbedrohliche Zustände. Stets muss bedacht werden, dass periphere Durchblutungsstörungen das Infektionsrisiko erhöhen und den Erhalt der Extremität gefährden. In solchen Fällen hat die konservative Behandlung ihren Stellenwert, eventuell mit Einsatz einer Extension.

\section{OP-Technik}

Der Eingriff erfolgt in Rückenlage, falls erforderlich und vertretbar in Blutsperre. Die dreifache Hautdesinfektion bezieht den Beckenkamm mit ein. Danach erfolgt die sterile Abdeckung. Abhängig von der
Bruchform und den betroffenen Gelenkanteilen wird der Zugang gewählt. Bei den meist bestehenden lateralen Brüchen wählt man einen anterolateralen Zugang (Abb.5). Nach Ablösen der Fußextensoren vom Schienbein kann das Gelenk unterhalb des Meniskus über einen queren Schnitt eröffnet werden. Der Meniskus und sein Aufhängeapparat müssen geschont werden. Zur Wiederherstellung der Gelenkfläche werden die Fragmente angehoben. Hierzu kann die laterale Kortikalis gefenstert werden und die Gelenkfläche mit Stößeln nach oben geschoben werden. Das Repositionsergebnis kann temporär mit Kirschner-Drähten gesichert werden. Nach Spongiosaplastik wird dann die definitive Osteosynthese vorzugsweise mit einem winkelstabilen Implantat vorgenommen. Hierbei müssen nicht zwingend alle Plattenlöcher winkelstabil besetzt werden. Wichtig sind die subchondral zu platzierenden proximalen Schrauben.

Zusätzlich zur visuellen Kontrolle des Repositions- und Operationsergebnisses kann man eine dreidimensionale Rekonstruktion mit einem 3D-Bildverstärker vornehmen (Abb. 6). Unmittelbar danach kann man noch in gleicher Sitzung etwaige Korrekturen durchführen. So können frühe Korrektureingriffe unter Umständen vermieden werden, was von Vorteil für den Unfallverletzten ist.

Bei fehlender medialer Abstützung ist eine alleinige Versorgung von lateral nicht ausreichend. Dies gilt auch bei Verwendung von winkelstabilen Implantaten. 

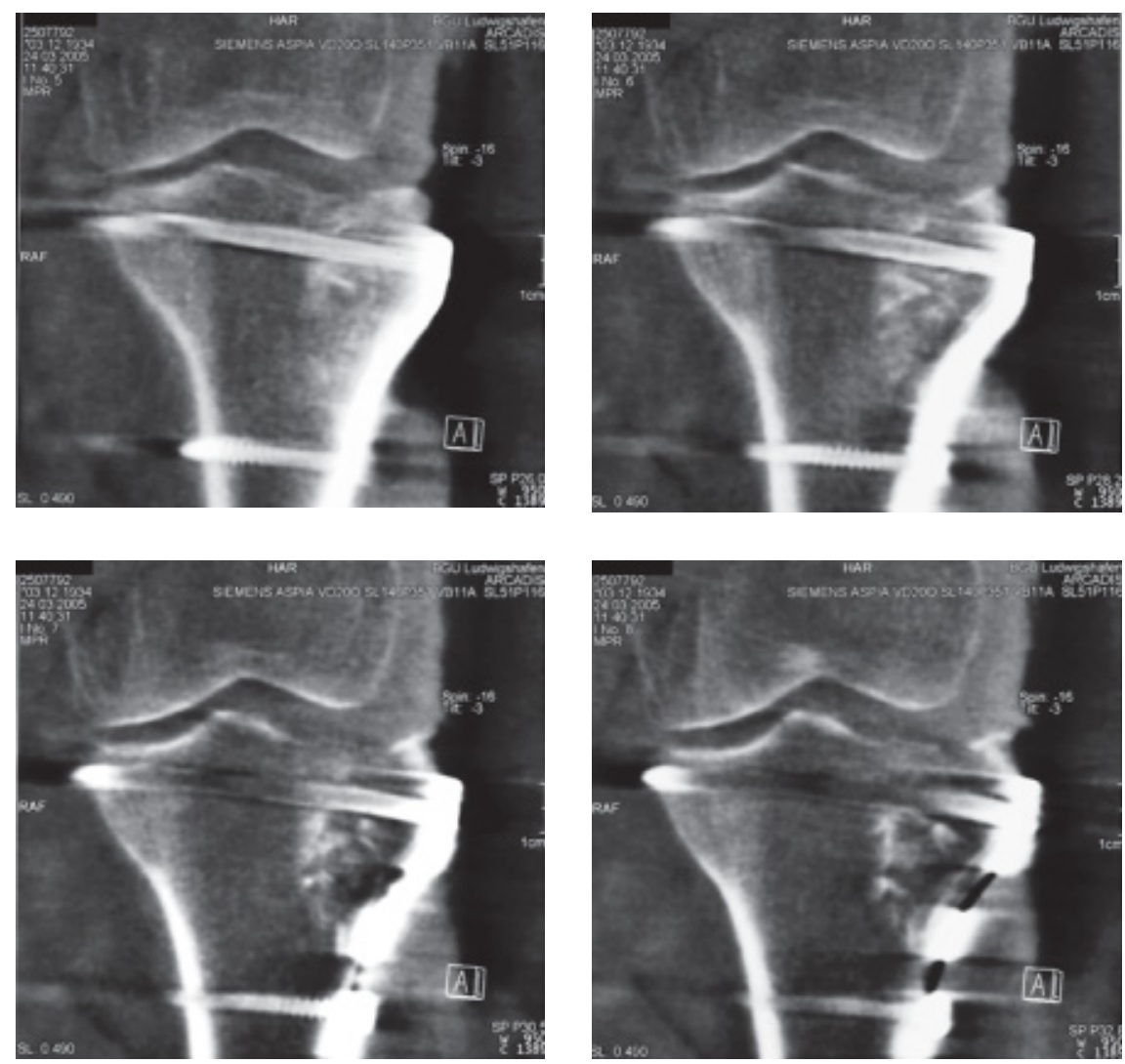

Abb. 6 intraoperativer 3D-Scan zur Beurteilung der Gelenkflächenrekonstruktion mit anatomischer Wiederherstellung. Gut erkennbar die eingebrachte Spongiosa lateral und die Balkenfunktion der unterhalb der Gelenkfläche eingebrachten winkelstabilen Schrauben.

Man sollte dann einen Fixateur externe von medial anbringen und vier bis sechs Wochen belassen. Von einer Doppelplattenosteosynthese muss man aufgrund des hohen Risikos für sekundäre Weichteilschäden dringend abraten. Allenfalls kommt eine minimal invasive Plattenosteosynthese von medial in Frage.

Der Wundverschluss erfolgt nach Gelenkspülung und Refixation des Meniskus über einer Drainage im Plattenlager. Die Faszie bleibt offen.

Spaltbrüche ohne Impression können nach Reposition mit einer transkutan eingesetzten spitzen Repositionszange durch subchondrale Spongiosaschrauben mit Unterlegscheiben minimalinvasiv gefasst werden. Die Reposition wird mit gelenkparallelen Führungsdrähten gesichert. Die Arthroskopie hat hier ihren Stellenwert.

\section{Nachbehandlung}

Bei Spaltfrakturen ist prinzipiell eine Teilbelastung von $20 \mathrm{~kg}$ über sechs Wochen möglich. Bei Impressionsbrüchen sollte über den gleichen Zeitraum nur Bodenbelastung gestattet werden. Der Belastungsaufbau wird nach der zwölften Woche abgeschlossen. Diese eher theoretischen Überlegungen können aber von den multimorbiden Patienten oft nicht umgesetzt werden.

Bei eingeschränkter Koordination wird die Teilbelastung nicht eingehalten.

Man behilft sich dann mit Gehböcken und Gehwagen. Es werden keine Bewegungslimitierungen vorgegeben, abgesehen von der Belastungseinschränkung ist eine funktionelle Nachbehandlung möglich.

\section{Ergebnisse}

Trotz der mit dem Alter einhergehenden Begleiterkrankungen sind nicht zwangsläufig Wundheilungsstörungen oder Pseudarthrosen zu erwarten. Man muss aber wie bei den Schenkelhalsfrakturen einen negativen Effekt der organischen und psychischen Erkrankungen unterstellen. Natürlich muss man bei den tendenziell kranken Unfallverletzten von einem erhöhten perioperativen Risiko mit entsprechenden Komplikationen ausgehen (Abb. 7).

Selbst bei optimaler Versorgung ist das Risiko einer posttraumatischen Arthrose bei u.a. Auftreten eines Korrekturverlusts hoch (Abb. 8). Die dadurch entstehenden Beschwerden führen zu einer Aktivitätseinbuße mit Beeinträchtigung der Mobilität und Eigenständigkeit.

Man sollte daher trotz der zu erwartenden Gonarthrose keine Kompromisse bei der Versorgung machen.

\section{Komplikationen}

Neben dem gefürchteten Kompartmentsyndrom können behandlungswürdige Gefäßverletzungen auftreten. Die Arthrose und der sekundäre Korrekturverlust enden je nach Aktivität und Leidensdruck in einem künstlichen Gelenkersatz. Es wird daher die primäre Knieprothese als Alternative zur Osteosynthese diskutiert [7]. Eine Komplikation mit möglicherweise fatalen Folgen ist der postoperative Infekt.

\section{Schlussfolgerung}

Durch die veränderte Alterstruktur werden in den kommenden Jahrzehnten mehr Rentner als Beschäftigte das Bild unserer Gesellschaft prägen. Das muss zwangsläufig zu einer erhöhten Anzahl an alterstypischen Brüchen führen. Gleichzeitig werden aber auch Frakturen zunehmen, die bisher überwiegend bei jungen Patienten im Rahmen von Hochrasanztraumen anzutreffen waren. Ein Beispiel hierfür ist der durch Osteoporose

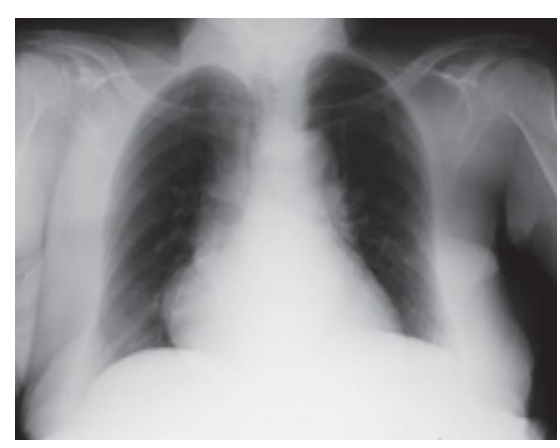

Abb.7 Röntgenthorax einer 75-Jährigen mit Linksherzinsuffizienz, Zeichen des Lungenemphysems, Aortenverkalkung und -elongation. An weiteren Begleiterkrankungen besteht eine unklare Anämie, eine bekannte Polyarthritis, multiple Allergien und ein chronisch venöses Ulkus am linken Unterschenkel mit Lymphödem. 

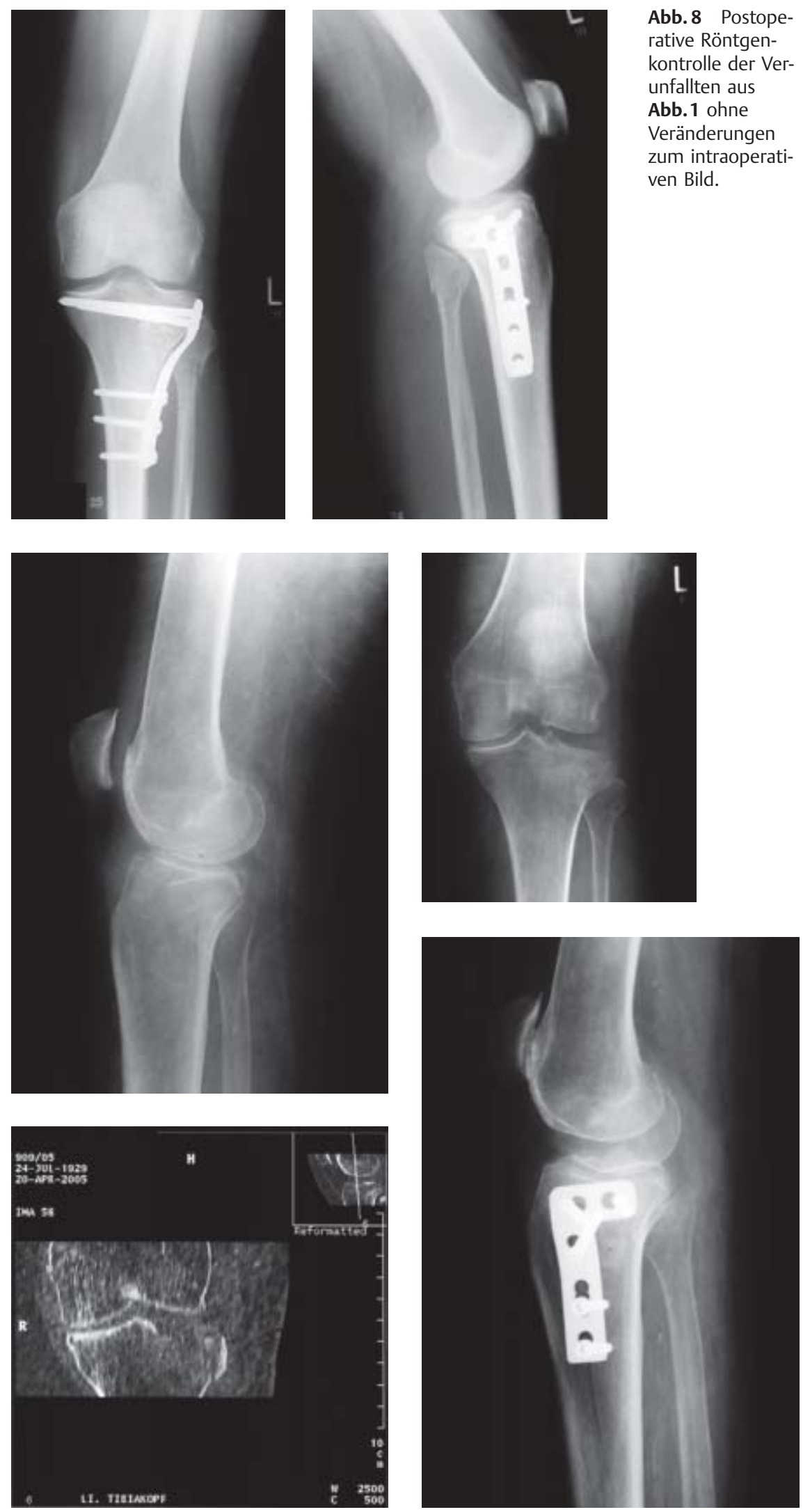

Abb.9 Röntgenverlauf der Unfallverletzten aus Abb. 7, die sich bei einem häuslichen Sturz neben der Tibiakopffraktur auch eine subkapitale Humerusfraktur zugezogen hat. Der stationäre Aufenthalt erstreckte sich wegen schwieriger Mobilisation und Wundheilungs-

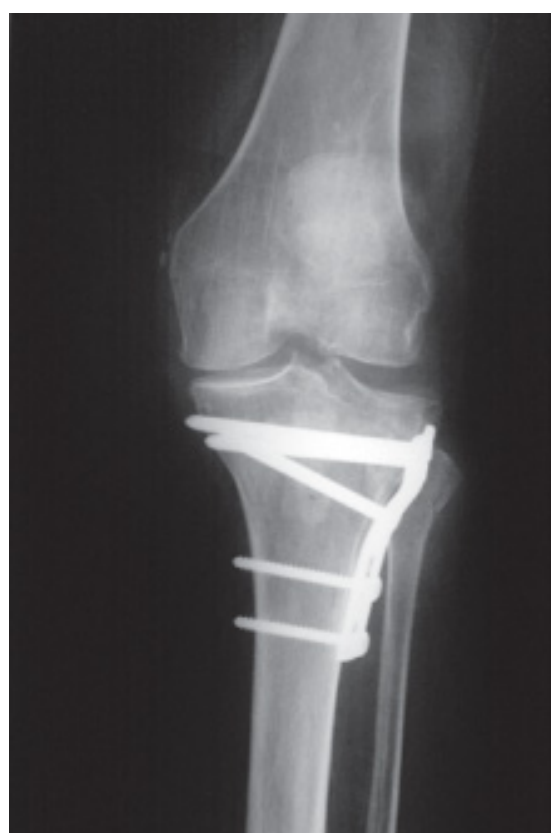

Abb. 8 Postoperative Röntgenkontrolle der Verunfallten aus

Abb. 1 ohne Veränderungen zum intraoperativen Bild.

störung durch ein vorbestehendes Lymphödem auf 28 Tage bis zur Verlegung in eine Kurzzeitpflegeeinrichtung mit der Möglichkeit einer direkt nachfolgenden Anschlussheilbehandlung. Eine Unterbringung in der Familie war aus verschiedenen Gründen nicht möglich. 
die Wiedereinbindung in das persönliche Umfeld.

Auch der alte Mensch will wieder schnell zurück in seinen Alltag finden. Arbeit ist hier gleichzusetzen mit der Bewältigung des Alltags. Jeder Funktionsverlust kann dabei zur Einengung des persönlichen Freiraumes und zum Verlust sozialer Kontakte führen. Auch die Angst vor Isolation spielt eine große Rolle. Ziel der weiteren Überlegungen hinsichtlich der künftigen geriatrischen Unfallchirurgie muss es daher sein, die altersgemäße Behandlung von sich herauskristallisierenden Verletzungen wie die Tibiakopffraktur nach Bagatellveletzung festzulegen. Eine rasche, komplikationsarme Wiederherstellung des alten Patienten gelingt dabei nur im Zusammenspiel mehrerer Disziplinen.
Außerdem sollte man diesen Verletzungen im Alter präventiv begegnen [1] Hierzu gehört eine suffiziente Osteoporosetherapie und die Abklärung der Sturzursache (Fehlsichtigkeit, Stolperfallen in der Wohnung oder sedierende Medikamente) [8].

Die volkswirtschaftliche Verantwortung, die der Arzt bei der Behandlung dieser Altersgruppe übernimmt, steht in krassem Gegensatz zur betriebswirtschaftlichen Situation. Die ökonomischen Zwänge des DRG-Systems lassen kaum Spielraum in der Behandlung dieser Altersgruppe. Ein kostendeckendes Arbeiten scheint bei längeren Verweildauern, der behandlungsintensiven Multimorbidität und den teureren winkelstabilen Implantaten nicht möglich (Abb.9).

\section{Literatur}

${ }^{1}$ Bartl R, Bartl C, Mutschler W. Diagnostik und Therapie der Osteoporose. Strategie für eine effiziente Prävention von Folgefrakturen. Unfallchirurg 2003; 106: 526-541

${ }^{2}$ Bengner U, Johnell O, Redlund-Johnell I. Increasing incidence of tibia condyle and patella patella fractures. Acta Orthop Scand 1986; 57: 334-336

${ }^{3}$ Ewerbeck V, Wentzensen A. Standardverfahren in der operativen Orthopädie und Unfallchirurgie. Thieme, Stuttgart 2003

${ }^{4}$ Gerich T, Blauth M, Witte F, Krettek C. Die Osteosynthese von Tibiafrakturen im höheren Lebensalter. Unfallchirurg 2001; 104 : $50-56$

${ }^{5}$ Gerich T, Bosch U, Schmidt E, Lobenhoffer P, Krettek C. Kniegelenksendoprothetik nach Tibiakopffrakturen. Mittelfristige Ergebnisse einer Kohortenanalyse. Unfallchirurg 2001, 104: 414-419

${ }^{6}$ Hansis M. Bsondere Aspekte bei der Behandlung von Verletzungen alter Menschen: epidemiologische, pathophysiologische, klinische Aspekte. OP-Journal 1995; 11: 5-8

${ }^{7}$ Kilian U. Kniegelenkendopprothese zur primären Therapie der intraartikulären Tibiakopffraktur im höheren Lebensalter. Unfallchirurg 2003 106: 1046-1051

${ }^{8}$ Runge M, Rittweger J, Felsenberg D. Das Sturzrisiko ist schnell kalkuliert. Ärztezeitung 2004; 110: 12

${ }^{9}$ Schirmacher F. Das Methusalem-Komplott. Karl Blessing, München 2004

${ }^{10}$ Siebert HR, Beck A. Unfallchirurgie im Alter Unfallchirurg 2005; 76: 139-150

\section{Dr. med. Christian Frank}

Chirurg

Priv.-Doz. Dr. med.

Paul Alfred Grützner

Oberarzt

Prof. Dr. med. Andreas Wentzensen Ärztlicher Direktor

BG-Unfallklinik Ludwigshafen

Ludwig-Guttmann-Str. 13

D-67071 Ludwigshafen 